\title{
DESIGN, DEVELOPMENT AND VALIDATION OF AN IMPACT WEAR TESTING DEVICE FOR THE MINERALS INDUSTRY
}

\author{
Lokesh K. Thakur \& Peter Radziszewski \\ Mechanical Engineering Department \\ McGill University, Montreal, Quebec H3A 2K6 \\ e-mail : lokesh.thakur@mail.mcgill.ca
}

\begin{abstract}
Total steel media wear in a given mill (ball or SAG) grinding process is a product of three recognized wear mechanisms-impact, abrasion, and corrosion of which the contribution of each wear mechanism to total media wear has not been well established. A total media wear model can be defined on the assumption that the effect of each wear mechanism can be independently determined and this effect can be tied to mill charge motion as determined or estimated using a charge motion simulator, which allows for a total media wear model to be defined as the summation of the wear results of each mechanism. This necessitates the need for developing impact, abrasion and corrosion wear testers that will allow the study of media wear over a wide range of energy levels. Refinement of the impact test will allow studying how impact media wear at high energies behaves as a function of those energies. This work is focused on design and development of a high-energy impact wear tester. A comprehensive illustration on designing the newest version of the impact wear tester is explained in detail in order to exhibit how theoretical, virtual and experimental analyses could be integrated while designing a mechanical system.
\end{abstract}

\section{Introduction}

In mineral processing industries, grinding processes can be defined as pulverization or comminution of big particles in order to reduce them to minute particles. Depending upon the mining operation, half of the overall operating cost of comminution processes can be found in the cost of steel media wear. Metal wear is primarily associated with these processes. Grinding performance and quality can also be affected by wear, which consists of three recognized wear mechanisms-abrasion, corrosion and impact.

In such a context, predictive wear models have become a necessity to determine most optimum grinding conditions that can reduce process wear while maintaining grinding performance and quality [1]. These impact and abrasion energy spectra provide an opportunity to study media wear as a function of the operating parameters of a given mill $[2,3]$. A tumbling mill, whether it is autogenous, semiautogenous, ball or rod, is a system comprises of a number of interrelated and interactive elements that work together in order to grind a given ore. This comminution process is achieved by individual balls that compose actual ball mill elements, which bring about ore breakage [4]. Jointly, these balls form the mill ball charge which, during ball mill operation, typically has a charge profile as can be found in the figures 1.

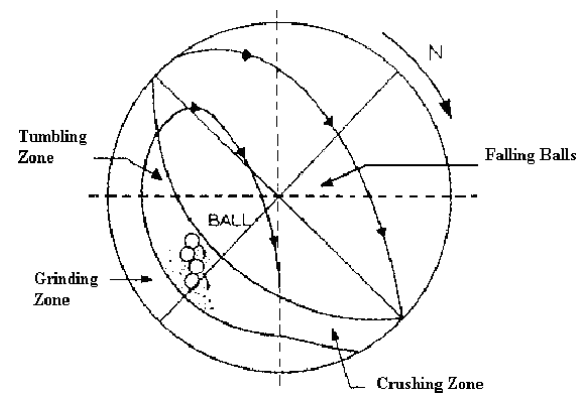

Figure 1: Typical ball charge motion profile.

A ball charge motion profile shows three comminution zones (figure 1) that can be distinguished by (i) ball layers sliding over one another grinding material trapped between them, the grinding zone, (ii) balls rolling over one another breaking material in low energy impact, the tumbling zone and by (iii) ball in flight re-entering into the ball charge crushing material in high energy impact, the crushing zone.

A total media wear model can be defined on the assumption that the effect of each wear mechanisms can be independently determined and this effect can be tied to mill charge motion as determined or estimated using a charge motion simulator which allows for a total media wear model to be defined as the summation of the wear result of the wear each mechanism [5].

$$
\dot{m}_{\text {total }}=\sum_{i=1}^{3} \dot{m}_{i}
$$


where $i=1,2,3$ represents the abrasion, corrosion and impact wear mechanisms respectively.

It is assumed that the contribution of each wear mechanism in equation 1 can be determined using the impact/abrasion energy spectra along with the lab test results. These lab tests were developed from some existing tests.

Abrasive Wear: The abrasive wear characteristics, as a function of applied abrasion force, were determined using steel-on-steel abrasion wheel test where the abrasive was a ground ore.

Corrosive Wear: The raw corrosion wear characteristics were determined from a batch test using the same ground ore and mill water in order to replicate a similar corrosion environment.

Impact Wear: Tumbling and free falling grinding media explain the phenomena of impact associated with the energies dissipated in tumbling and crushing zones of the ball charge motion profile (figure 1). Three impact testers found in the literature $[6,7,8]$ present promising aspect for determining grinding media impact wear. The impact chamber principle shown by the Scieszka and Dutkiewicz tester [8] presented the most flexible impact wear/impact energy results for different ore-metal-environment combinations. Based on these analyses, it is possible to reformulate the equation 1 for a similar ore-metal-environment industrial context to,

$$
\begin{aligned}
m_{\text {total }}= & \sum_{i=1}^{n_{a b r \text { int }}} m_{a b r i}\left(E_{a b r i}\right) n_{a b r i}+\frac{m_{2}}{A_{l \text { ball }}} A_{r \text { ball }} \\
& +\sum_{j=1}^{n_{\text {imp int }}} m_{i m p j}\left(E_{\text {imp j }}\right) n_{\text {imp } j}
\end{aligned}
$$

\subsection{Exploring Impact Media Wear: Semi- empirical Approach}

The basic objective of the impact tester is to determine impact wear for different media types as a function of the energies involved in impact.

Impact wear per ball $m_{3}$ can be described using the adhesion model for wear as a function of energy dissipated in impact $E_{3}$, metal hardness $H_{r}$, metal density $\rho$ and a wear probability constant $P_{c}[2,3,8,9]$ :

$$
m_{\text {impact }}=\rho \frac{P_{c}}{3 H_{r}} E_{\text {impact }}
$$

As mentioned in the P9L study on exploring total media wear [10], it has been observed that the laboratory set-up although allowing impact variation, did not attain the desired impact energy levels.

As the impact chamber can not generate impact energies greater than that equal to a $4 \mathrm{~cm}$ drop, a more aggressive test must be developed. A new impact tester has been developed that would generate impacts in the order of those found in the USBM media drop test [11]. The design criteria for the newer test is flexibility in impact energies and impact velocities. This has led to the development of a precompressed spring cam assembly that can be schematized as in the figure 2 .

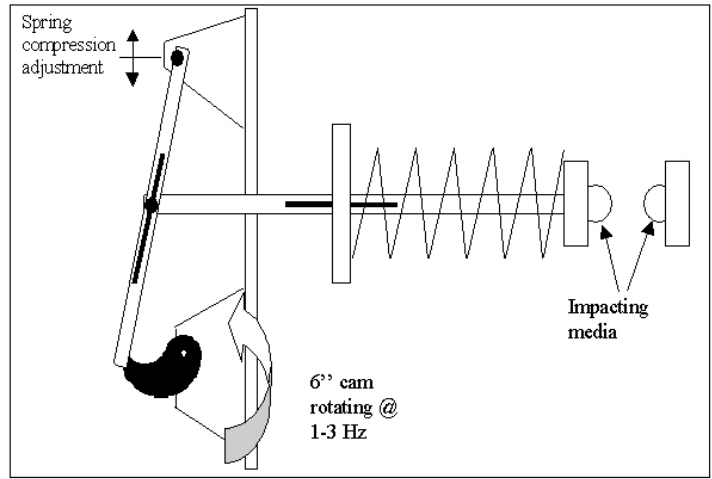

Figure 2: High energy impact test mechanism schematic [11].

\section{High Energy Impact Wear Tester Development}

The figure 3 shows a 3-D model of the version I of the impact tester prototype. As shown in this figure, the caret holder is assembled to the double threaded cylinder and this cylinder is assemble to the spring shaft assembly while this spring shaft assembly is connected to the Lever arm assembly. The spring is inside the stationary spring housing with one end sitting against the stationary plate and the other end in contact with the reciprocating double threaded cylinder.

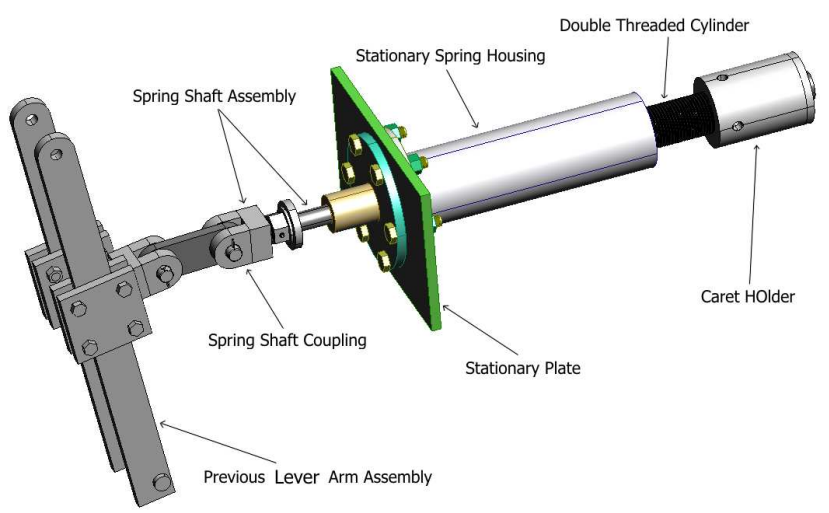

Figure 3: Version I of the impact tester assembly [12].

Initial Test Results: A few tests were carried on the version I of the high energy impact tester in order to vali- 
date its performance against predefined set of goals, which were exclusively focused on achieving maximum speed at the impact. High resolution slotted optical switches were used for measuring speed at the impact. Following assumptions taken during Observations [12]: (1) the system was assumed to be a frictionless system and (2) acceleration gradient was assumed to be equal to zero.

A medium pressure die spring (color coded blue) with a spring constant $(\kappa)=29.427 \mathrm{~N} / \mathrm{mm}$ [13] was used. The coupling was positioned on the vertical arm in such way that the spring compression within the efficient operating range (25 $\%-35 \%$ of free length) could be achieved [13]. The spring was compressed for approximately 4 " or $101 \mathrm{~mm}$, which is $33.33 \%$ of its free length (12" or $304.8 \mathrm{~mm}$ ). This version of the high energy impact could only produce impact energy equivalent to $9.8 \mathrm{~cm}$ of drop height. Following errors were found in the results: (1) optical switches were very sensitive to high amplitude of vibrations produced by the mechanical system during its operation and (2) electronic counter couldn't be calibrated properly, this is why its readings could have had moderate degree of error.

Because of above mentioned errors in the observation, optical switches couldn't be used for the further measurements. This is why a high-speed camera was used for measuring speed at the impact while running tests on the version II of the high energy impact wear tester.

\section{Theoretical and Virtual Dynamics of the Impactor Mechanism}

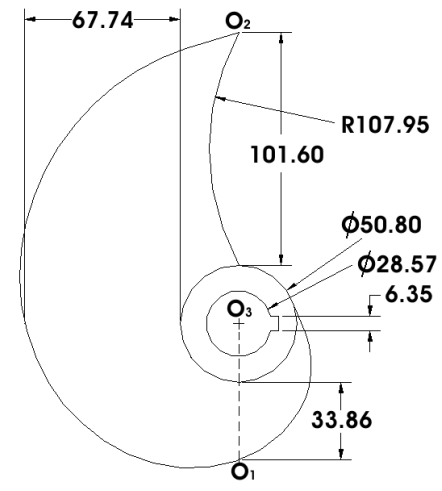

Figure 4: Cam profile in $\mathrm{mm}[14]$.

As shown in the figures 4 and 5, contact point at the follower (location $\mathrm{O}_{1}$ ) rotates at a radius of $\mathrm{r}_{\max }$ from the pivot point $\mathrm{O}$. The contact point at the cam (location $\mathrm{O}_{1}$ ), which is situated at a distance of $\mathrm{r}_{\max }$ from the pivot point $\mathrm{O}_{o}$, rotates from the initial value of radius $(59.20 \mathrm{~mm})$ to the maximum value of radius (6" or $152.40 \mathrm{~mm}$ ). After leaving the cam (location $\mathrm{O}_{2}$ ), follower moves rapidly in the

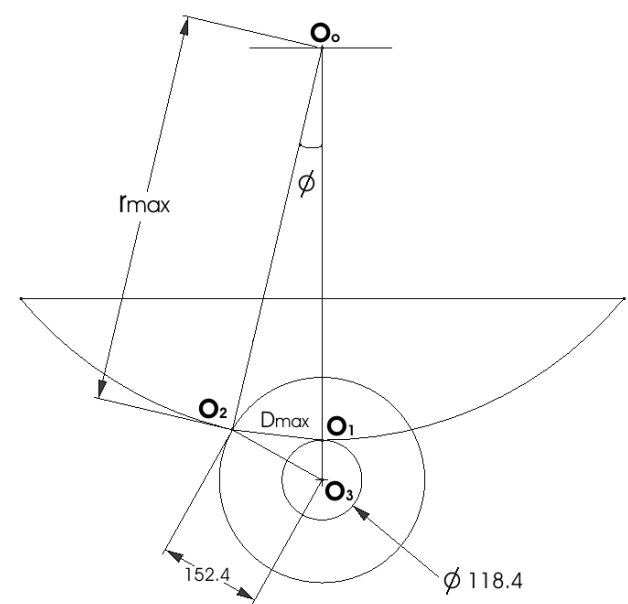

Figure 5: Path traced by cam-follower contact point [12].

forward direction along with the Lever arm assembly under the influence of potential energy delivered by the compressed spring. This assembly moves until the caret, which is assembled at the forefront of mobile assembly, collides with the stationary target ball. As shown in the figure 5, the cam-follower contact point moves back and forth between locations $\mathrm{O}_{1}$ and $\mathrm{O}_{2}$.

\subsection{Theoretical Dynamics of the System}

Derivation for Calculating Spring Displacement: In the triangle $\mathrm{O}_{0}, \mathrm{O}_{3}$ and $\mathrm{O}_{2}$ (figure 5) $\angle \phi\left(\angle \mathrm{O}_{2} \mathrm{O}_{0} \mathrm{O}_{3}\right)$ can be calculated as [15],

$\left(O_{3} O_{2}\right)^{2}=\left(O_{2} O_{0}\right)^{2}+\left(O_{0} O_{3}\right)^{2}-2\left(O_{2} O_{0}\right)\left(O_{0} O_{3}\right) \cos \phi$

For a small angular displacement, in the triangle $\mathrm{O}_{0}, \mathrm{O}_{1}$ and $\mathrm{O}_{2}, D_{\max }$ or maximum displacement of the spring for a radius $r_{\max }$ (figure 5) can be calculated as [15],

$$
D_{\max }=2 \sin (\phi / 2) r_{\max }
$$

Since the value of $\angle \phi$ is constant for a given set of lengths therefore a formula can be derived as [12],

$$
D_{i}=k r_{i} \quad i=1,2,3, \ldots
$$

where $r_{i}$ is a variable radius defined as the distance between coupling axis and the axis of rotation, which passes through the pivot point $\mathrm{O}_{0}$ and $k$ is a constant, which depends on dimension of the Lever arm and the cam [12].

Derivation for Calculating Speed at the Impact: Equation for calculating speed at the impact can be derived from law of conservation of energy. In case of reciprocating impact mechanism potential energy stored in the spring media will be delivered to the reciprocating mass and the Lever arm, out of which some amount of energy will be lost 
in overcoming the friction. Potential energy stored in the spring can be expressed as,

$$
E_{p s}=\frac{1}{2} \kappa x^{2}
$$

where, $E_{p s}$ is the potential energy stored in the spring in $\mathrm{Nmm}$ or $\mathrm{Nm}$. This energy will be converted into momentum energy and some part of it will be lost in overcoming the friction. Momentum energy $\left(E_{m r c}\right)$ for the reciprocating components can be expressed as,

$$
E_{m r c}=\frac{1}{2}\left(W_{r c}^{\prime}\right) v_{i}^{2}
$$

where,

$v_{i}=$ Speed at the impact in $\mathrm{m} / \mathrm{sec}$.

$W_{r c}^{\prime}=$ Total weight of the reciprocating components in $\mathrm{kg}$.

Momentum energy $\left(E_{m v c}\right)$ for the rotating components (Lever arm assembly) can be expressed as [12],

$$
E_{m v c}=\frac{1}{2}\left(W_{l a}^{\prime} \cos \phi\right) v_{i}^{2}
$$

where,

$W_{l a}^{\prime}=$ Weight of the Lever arm assembly in $\mathrm{kg}$.

$\phi=$ Angular displacement of the Lever arm in radians.

As we can see from the equation 9 , only vertical component $\left(W_{l a}^{\prime} \cos \phi\right.$ ) of the Lever arm assembly is considered due to the fact that only this component would oppose the motion. Horizontal inertial component of the Lever arm assembly will help the spring to restore its original length, therefore momentum energy $\left(E_{m h c}\right)$ associated with this component will be added to the input energy to the system, it can be expressed as [12],

$$
E_{m h c}=\frac{1}{2}\left(W_{l a}^{\prime} \sin \phi\right) v_{i}^{2}
$$

Part of the input energy lost in overcoming the friction $\left(E_{f v a}\right)$ for the Lever arm assembly can be expressed as [12],

$$
E_{f v a}=\mu_{d r} W_{l a}^{\prime} \cos \phi\left(\phi r_{p}\right)
$$

where,

$\mu_{d r}=$ coefficient of dynamic rolling friction for the rotating mass (Lever arm), and

$r_{p}=$ radius of the pivot pin about which Lever arm is hanged in $\mathrm{mm}$, which is equal to $5 / 16$ " or $7.94 \mathrm{~mm}$.

Part of the input energy lost in overcoming the friction $\left(E_{f r c}\right)$ for the reciprocating components assembly can be expressed as [12],

$$
E_{f r c}=\mu_{d s} W_{r c}^{\prime} D_{i}
$$

where,

$\mu_{d s}=$ coefficient of dynamic sliding friction for the reciprocating components assembly, and
$D_{i}=$ displacement or compression amplitude of the spring in $\mathrm{mm}$.

Now, an expression for calculating speed at the impact can be written as [12],

$$
E_{p s}+E_{m h c}=E_{m r c}+E_{m v c}+E_{f v a}+E_{f r c}+E_{a b s}
$$

where $E_{a b s}$ is the maximum energy absorbed by the Lever arm during loading conditions, which is equal to $1 / 2 F f_{\max }$. Where $\mathrm{F}$ is the load on the Lever arm in $\mathrm{N}$ and $f_{\max }$ is the maximum deflection of it under the influence of force $\mathrm{F}$ in $\mathrm{mm}$. The equation 13 can be rewritten as,

$$
\begin{aligned}
E_{p s} & +\frac{1000}{2}\left(W_{l a}^{\prime} \sin \phi\right) v_{i}^{2}=\frac{1000}{2}\left(W_{r c}^{\prime}+W_{l a}^{\prime} \cos \phi\right) v_{i}^{2} \\
& +\mu_{d r} W_{l a}^{\prime} \cos \phi\left(\phi r_{p}\right)+\mu_{d s} W_{r c}^{\prime} D_{i}+E_{a b s}
\end{aligned}
$$

For a particular set of conditions i.e., spring constant, spring displacement etc. $x$ and $D_{i}$ would be equal, therefore equation 14 can be rewritten as,

$v_{i}=\sqrt{\frac{\kappa D_{i}{ }^{2}-2\left[\mu_{d r} W_{l a}^{\prime}\left(\phi r_{p}\right) \cos \phi+\mu_{d s} W_{r c}^{\prime} D_{i}-E_{a b s}\right]}{1000\left[W_{r c}^{\prime}+W_{l a}^{\prime} \cos \phi-W_{l a}^{\prime} \sin \phi\right]}}$

\subsection{Virtual Dynamics of the System}

A virtual prototype was built using a very powerful virtual prototyping simulation software know as ADAMS (Automatic Dynamic Analysis of Mechanical Systems).

Virtual Modelling Parameters: Simulations were run for 2.5 seconds with a total of approximately 3400 frames in order to simulate like a very precise high speed camera with approximately 1400 frames/sec. The strongest spring $(\kappa=$ $124.717 \mathrm{~N} / \mathrm{mm}$ ) with a compression amplitude of $95.80 \approx$ $96.00 \mathrm{~mm}$ was modelled for all the simulation runs in order to simulate the toughest testing conditions [12].

The First Simulation Run: In the first simulation run, full weight of all the moving components and a value of 0.35 [17] as a friction coefficient for the Lever arm pivot as well as for the reciprocating components were simulated. From the simulation, maximum speed at the impact was found to be equal to $4.5 \mathrm{~m} / \mathrm{sec}$. The table 1 shows weight of all the moving components measured from version I of the impact tester.

\begin{tabular}{|c|c|c|}
\hline S. No. & Component Name & Weight (kgs) \\
\hline \hline 1 & Caret Holder with Caret & 1.9 \\
\hline 2 & Double Threaded Cylinder & 2.9 \\
\hline 3 & Spring Shaft Assembly & 4.0 \\
\hline 4 & Vertical Arm Assembly & 12.3 \\
\hline
\end{tabular}

Table 1: Weight of moving components from version I [12]. 
The Second Simulation Run: In the second simulation run, weight of all the moving components and friction coefficient (by assuming proper lubrication and improvement in the design) were halved except weight of the Lever arm assembly, which was reduced to one forth of its original value. The maximum speed at the impact in $\mathrm{m} / \mathrm{sec}$ was found to be equal to $11.26 \mathrm{~m} / \mathrm{sec}$.

Verification of the Virtual Model: If maximum radius of the cam is $R_{\text {cam }}$, an expression for calculating maximum torque $\left(T_{\max }\right)$ delivered by the cam under equilibrium condition can be written as [12],

$$
T_{\max }=\frac{r_{1}}{r}(\kappa x) \times R_{c a m}
$$

where $R_{\text {cam }}$ is the maximum radius of the cam, which is equal to 6 " or $152.40 \mathrm{~mm}$. The maximum value of torque calculated from the ADAMS simulation was $1375.14 \mathrm{Nm}$ and from the theoretical calculations was $1311.01 \mathrm{Nm}$. The virtual model had an error of $4.89 \%$, which is quite reasonable after considering the fact that errors are almost inevitable during the building of virtual prototype model from a physical prototype [12].

Virtual prototyping helped to know which variables are critical to the optimization. To define variables, we need to know which variables have an effect on the variable to be minimized. The objective was to minimize volume of the moving components which is directly related to weight of the components. Further, results from ADAMS simulations were used for determining variables for optimizing designs while using ANSYS for the finite element analysis purposes.

\section{Lever Arm Assembly Design}

The Lever arm assembly was designed in a logical design sequence in order to overcome the design problems faced during working with the previous Lever arm assembly of version I of the impact wear tester. Following problems were encountered during working with the previous Lever arm assembly of version I of the impact wear tester, as shown in the figure 6: (1) The assembly was very bulky (12.30 kgs). (2) It had 18 parts, which is a very large number. (3) It was hung at two pivot points. This kind of design increases friction. (4) It was very difficult to adjust the spring compression amplitude by lowering and raising the coupling on the Lever arm. Nine different evolutionary designs of the Lever arm assembly were proposed in order to remove aforementioned problems.

Figure 7 shows final design ( $9^{\text {th }}$ design) of the Lever arm assembly, which had following advantages over the previous Lever arm assembly (figure 6): (1) It has only 10 number of parts versus 18 number of part in the previous version (figure 6). (2) It hangs at only one pivot point, which offers less friction than the previous design (figure 6) during any

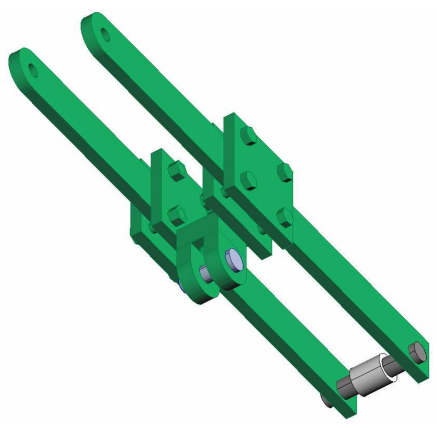

Figure 6: Previous Lever arm assembly.

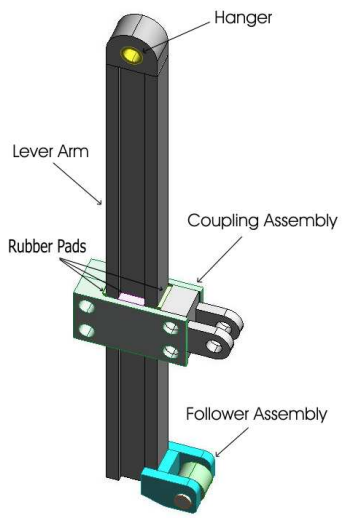

Figure 7: Lever arm assembly: final design [12].

movement. (3) Very easy to change the position of coupling on the Lever arm for altering spring compression amplitude. (3) Overall weight of it, if the same material used would be about $3.10 \mathrm{~kg}$ lighter than the previous Lever arm assembly design (figure 6).

Material Selection: Titanium ASTM Grade 5, Ti - 6Al - 4V (alpha-beta alloy) was chosen as the material to manufacture Lever arm assembly because of the following reasons [18]: (1) The most easily available and the most widely used titanium alloy. (2) It has very high strength and excellent fatigue properties. (3) The alloy is weldable. (4) It has very good mechanical properties up to $550^{\circ} \mathrm{C}$. (5) Its intrinsic resistance to corrosion practically eliminates maintenance in most environments. Ti-6Al-4V (ASTM Grade 5) has a fatigue strength of $490 \mathrm{MPa}$ for a smooth specimen $\left(K_{t}=1\right)$ under rotating bending condition for stress to cause failure in $10^{7}$ cycles [18].

Maximum Allowable Design Stress: The safety factor for structural systems, proposed Philon of Byzantium (3rd century BC) [19], is defined as follows

$$
N=\frac{\text { capacity }}{\text { load }}=\frac{\text { strength }}{\text { stress }}>1
$$

The value of a central safety factor (equation 17) should not be less than two (2) for most structural applications and 
should routinely be set at three (3). Uncertainty in loading, uncertainty in material properties, foreseeable abuse, and challenging service environments demand higher values of the safety factor. A long service life also requires a larger value of safety factor. High reliability applications require systems with a larger central safety factor value. Cyclic loading induces fatigue failure in structural components. The safety factor for primarily cyclic loading should be based on the endurance limit rather than on yield or tensile strength [20].

After taking consideration of uncertainties and assumptions associated with the overall reliability of the design; a value of three (3) as a FOS can reasonably be assumed. From the equation 17, maximum allowable design stress can be calculated as [12],

$$
\text { Design Stress }=\frac{490}{3}=163.33 \mathrm{MPa}
$$

where fatigue or endurance strength of the Titanium Grade 5, Ti - 6Al-4V (alpha-beta alloy) is 490.0 MPa [18]. As on results, the use of Titanium ASTM Grade 5 further lightens the weight of Lever arm assembly to approximately $5.0 \mathrm{~kg}$ [12].

\subsection{Theoretical Analysis: Plastic Design}

Early effort in stress analysis was based on limit loads, that is, a load which stresses a member "wholly" to the yield strength. More recently, the concept of limit loads, referred to as limit or plastic design has found strong application in the design of certain structures [21]. The theory presupposes a ductile material, absence of stress raisers, and fabrication free of embrittlement. Local load overstress is allowed, provided the structure does not deform appreciably. The problem is that of finding the final limit load. Elasticflexure theory gives the final limit load-triangular distribution as,

$$
F_{y}=\frac{2 S_{y} b h^{2}}{3 l}
$$

For the rectangular stress distribution, the limit load becomes

$$
F_{L}=\frac{S_{y} b h^{2}}{l}
$$

The ratio $F_{L} / F_{y}=1.50$ - an increase of 50 percent in load capability. The ratio $F_{L} / F_{y}$ has been named shape factor. The figure 8 shows shape factors for a typical I-section. The shape factor may also be determined by dividing the first moment of area about the neutral axis by the section modulus.

\section{Lever Arm Assembly Design: FEA}

In order to design Lever arm assembly components precisely, in the later stages of this assembly design, compu-

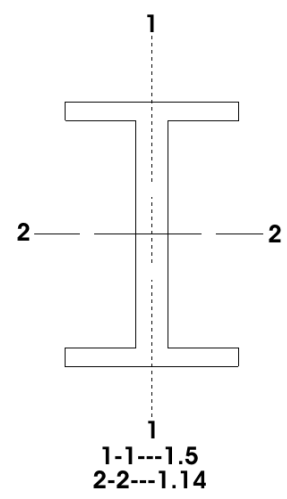

Figure 8: Shape factors for I-section [12].

tational finite element analyses (FEA) were used. ANSYS 7.0 (2002) was used comprehensively for this purpose.

\subsection{Lever Arm Design}

In the first stage of the Lever arm assembly design, Lever arm was designed because design of the coupling and the follower assemblies were dependent on its design.

A Model of the Problem: A finite element analysis (FEA) model was built on the assumption that the maximum force at the middle of the beam span will produce maximum deflection [12], which simulates the toughest physical condition scenario.

In the ADAMS simulation, the strongest spring was compressed to $96.00 \mathrm{~mm}$ of amplitude but for the finite element analysis, spring force was calculated for the 101.50 $\mathrm{mm}$ of compression amplitude in order to generate more confidence in the design because it is always advisable to a new designer that either use a fraction (90\%-95\%) of material's fatigue strength or use a greater value of the pressures or forces applied on the components [22].

Design Optimization: As mentioned earlier, the Lever arm basically is an I beam, which hangs about the pivot point $O_{0}$ (figure 5) at one end and attached to the follower at the other remaining end (figure 7). The vertical distance between these two points is $563.00 \mathrm{~mm}$, which is constrained by its frame. Therefore, the cross sectional dimensions were determined using ANSYS's built-in optimization tool.

Parameters for Design Optimization: Following parameters were set in order to run the design optimization tool in ANSYS [12]. (1) Design Variables (DVs): Initial approximation of the I beam cross section were set as the DVs for optimizing the design. (2) State Variable (SV): Maximum allowable stress in the beam was set as the SV, which was calculated as 163.33 MPa (equation 18). (3) Objective Variable (OV): Volume of the I beam was set as the OV.

Final I Cross Section: I beam cross section obtained by using optimization tool was expensive to manufacture be- 
cause Titanium's manufacturing cost is higher than the material cost itself. This is why a new I beam cross section was adapted according to the available Titanium sheets dimensions for reducing the manufacturing cost after consulting a material supplier.

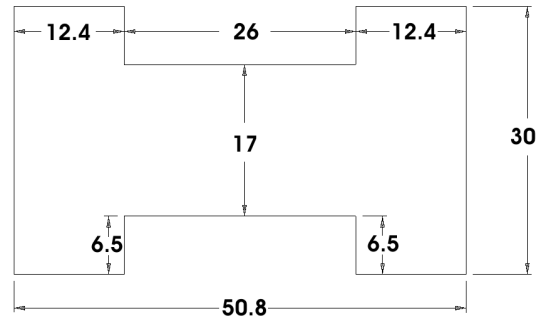

Figure 9: Finally adapted I beam section in mm [12].

The figure 10 shows results obtained from the final FEA for the I section showed in the figure 9 .

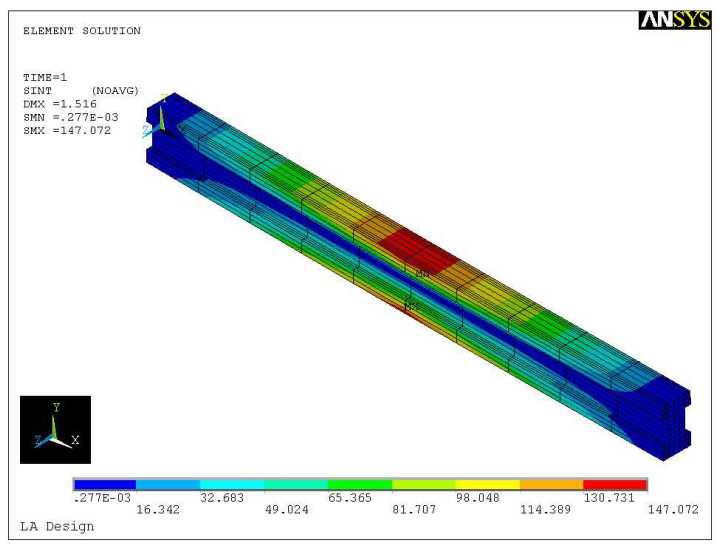

Figure 10: FEA of the finally adapted I beam section [12].

Validation of FEA Results with Theoretical Analysis: Lever arm design was used for ANSYS simulation validation purposes due to the fact that only the I beam analysis was carried out comprehensively at the theoretical level because of its regular structural geometry. And FEA was use to analyze designs of all the Lever arm assembly components comprehensively.

I beam properties were calculated for the I cross section shown in the figure 9. Design of beams may be based on strength (stress) or on stiffness if deflection must be limited. Maximum stress in MPa of an I beam can be calculated as,

$$
S_{\max }=\frac{M_{b_{\max }}}{I / c}=\frac{12700 \times 563 / 4}{308700.613 / 25.4}=147.078
$$

As it can be seen from the figure 10, maximum stress in the beam calculated from ANSYS is $147.072 \mathrm{MPa}$, which is very close to the value of maximum stress calculated theoretically (equation 21).

\subsection{Coupling Assembly Design}

The coupling assembly was designed, which consists of two side brackets and a front part (figure 7). Figure 11 shows a 3-D solid model of the coupling assembly (exploded view).

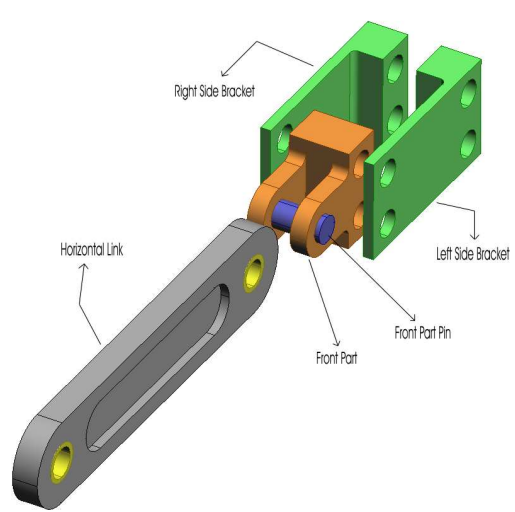

Figure 11: An exploded view of the coupling assembly [12].

Design of the Front Part: Boundary conditions for the front part were determined by assuming that the spring force would be transmitted to the part assembled in series. Which means that two front flanges of the front part would be under the influence of a force, tensile in nature and $12,700 \mathrm{~N}$ in magnitude [12].

Determination of Dimensions by Space Planning: A simple FEA was performed for determining thickness of the horizontal link. In order to reduce the manufacturing and the material cost associated with Titanium (Ti$6 \mathrm{Al}-4 \mathrm{~V})$, high quality regular structural steel with fatigue strength $=500 \mathrm{MPa}\left(10^{7}\right.$ cycles) [23] was used to manufacture the horizontal link (figure 11). It was found out that a thickness of $13.00 \mathrm{~mm}$ would be sufficient to ensure no failure $(\mathrm{FOS}=3.0)$ under the influence of $12,700 \mathrm{~N}$ of tensile force [12].

Several materials were considered for manufacturing the front part pin and the bolts, which could be used in assembling front part with the side brackets and the horizontal link (figure 11). It was found out that hex socket head shoulder screws of $5 / 8$ " or $15.88 \mathrm{~mm}$ diameter made from grounded and heat treated stainless steel have a minimum single shear strength of 28, $785 \mathrm{lbs}(128,070 \mathrm{~N})$ at its shoulder part [25].

A few thorough finite element analyses were performed in order to determine remaining dimensions of the front part. Following figure shows an FEA result from ANSYS for the finally designed front part.

Design of the Side Brackets: Space planning in conjunction with ANSYS FEA was used to reach the final design of the side brackets. Assuming the symmetrical distribution of the force exerted on the left and right side brackets due to the displacement of the spring, it can be stated that the force 


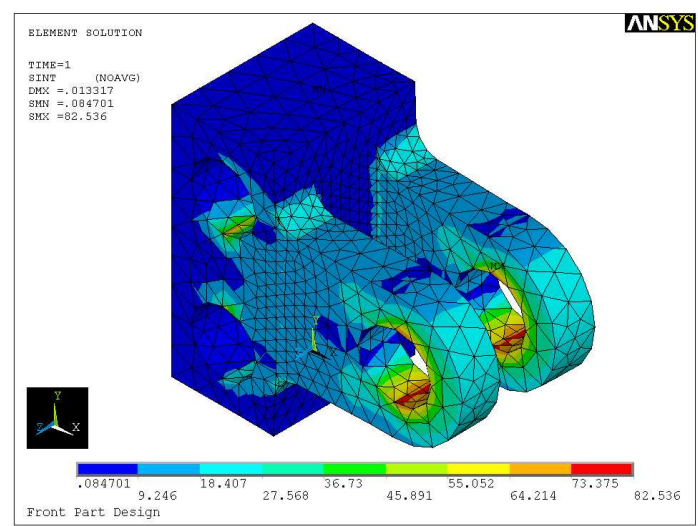

Figure 12: Front part design: final FEA [12].

will be equally distributed between them.

A few thorough finite element analyses were performed in order to determine remaining dimensions of the side brackets. Following figure shows an FEA result from ANSYS for the finally designed side bracket.

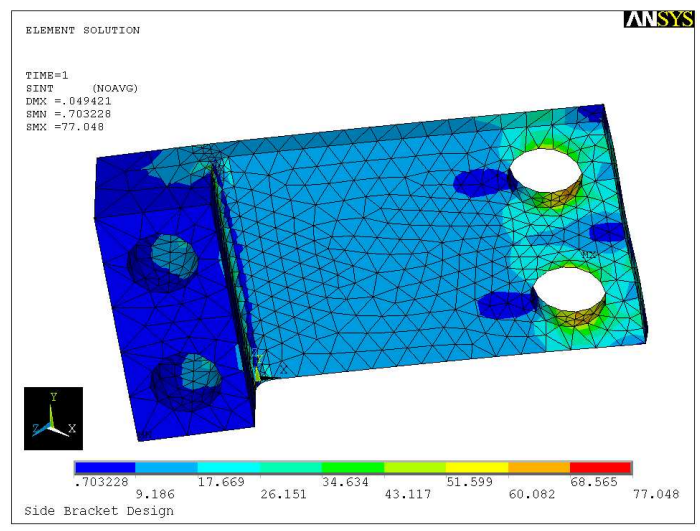

Figure 13: Side bracket design: final FEA [12].

\subsection{Follower Assembly Design}

The follower assembly consists of three components; main body, follower pin and follower roller. Except the main body, the follower pin and the follower roller were designed solely on the space available to assemble them and the cam thickness (figures 2 and 7). Stainless steel was chosen as a material to manufacture the follower roller because stainless steel is a standard material for manufacturing followers [24]. The cam was $22.00 \mathrm{~mm}$ in thickness, this is why in order to insure efficient use of the space available, the width of the follower roller was finalized at $22.00 \mathrm{~mm}$. Since Ti$6 \mathrm{Al}-4 \mathrm{~V}$ has excellent welding properties $[26,18]$ so it was decide that the follower assembly would be welded to the Lever arm. The figure 14 shows an exploded view of the follower assembly.

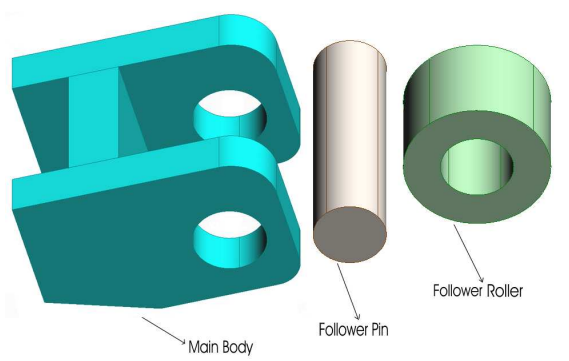

Figure 14: An exploded view of follower assembly [12].

While determining dimensions of the main body, a few interrelated parameters like outer diameter of the follower roller, its position on the main body and the main body's position on the Lever arm were determined in order to insure no contact between tip of the cam and the bottom part of the Lever arm, when the cam leaves the follower roller. A few trigonometrical geometries were sketched for determining the path traced by the cam-follower roller contact point. Later ADAMS simulation software in conjunction with ANSYS was used to simulate various position of the main body on the Lever arm in order to reduce stress concentration at the Lever arm-main body interface. It was found out that an outer diameter of $32.00 \mathrm{~mm}$ for the follower roller will help to insure no contact between the cam and the bottom part of the Lever arm [12].

A few thorough finite element analyses were performed in order to determine remaining dimension of the main body. The figure 15 shows an FEA result from ANSYS for the finally designed main body.

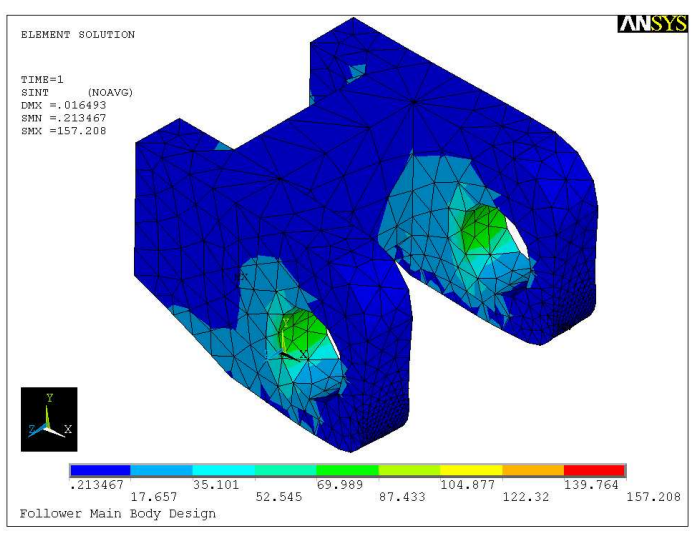

Figure 15: Main body design: final FEA [12]. 


\section{Development and Validation of Ver- sion II of the Impactor Prototype}

After having designed all the Lever arm assembly components, their engineering drawings were sent to Elimetal Inc. (a local manufacturer) to be manufactured by wire EDM method after consulting a manufacturing specialist [24].

In order to reduce weight of the reciprocating assembly further, which has a profound impact on speed at the impact; material of the spring shaft assembly and the double threaded cylinder was changed from regular structural steel to Al 7075-T6; a very high strength material used for highly stressed structural parts. Aluminum 7075-T6 is the strongest structural alloy of the aluminum's alloy family with density equal to $2.81 \mathrm{~g} / \mathrm{cc}$ or $2810 \mathrm{~kg} / \mathrm{m}^{3}$ [18]. A few simple FEA were performed using ANSYS in order to find out the maximum stress concentration in these components during loading conditions. It was found out that the maximum stress concentration would be less than the allowable design stress (53.33 MPa) for the FOS equal to 3.0 during the actual loading conditions based on fatigue strength of the material [18].

The table 2 shows the weights of mobile components of version II of the impact wear tester. In this table, the spring shaft assembly also includes weight of the horizontal link (figure 11). Here, percentage reduction is based on the weights of mobile components shown in the table 1.

\begin{tabular}{|c|c|c|}
\hline $\begin{array}{c}\text { Components } \\
\text { name }\end{array}$ & $\begin{array}{c}\text { Weights } \\
\text { in kgs }\end{array}$ & $\begin{array}{c}\text { \% } \\
\text { Reduction }\end{array}$ \\
\hline \hline Double Threaded Cylinder & 1.03 & 64.48 \\
\hline Spring Shaft Assembly & 2.60 & 35.0 \\
\hline Lever Arm Assembly & 5.12 & 58.37 \\
\hline
\end{tabular}

Table 2: Weights after upgrading impactor prototype [12].

The figure 16 shows an actual photograph taken after assembling the complete Lever arm assembly along with the horizontal link to the impact tester version II.

After having all the parameters related to the impact tester's dynamics e.g., dimensions and weights of the all the reciprocating components, the equation 6 for calculating spring displacement $\left(D_{i}\right)$ was modified [12]. The value of angle $\phi$ was calculated for the Lever arm posture shown in the figure 5, the equation 4 gives $\phi=13.21^{\circ}$. Now the equation 6 can be modified as,

$$
D_{i}=2 \sin (13.21 / 2) \lambda r_{i}=0.23 \lambda r_{i}
$$

where $\lambda$ is a correction factor, which was introduced after assembling the impactor prototype. Based on observations, a value of 0.735 was introduced as a correction factor.

Tests on the Impactor Performance: For impact tester's validation purposes, a high pressure die spring (color coded

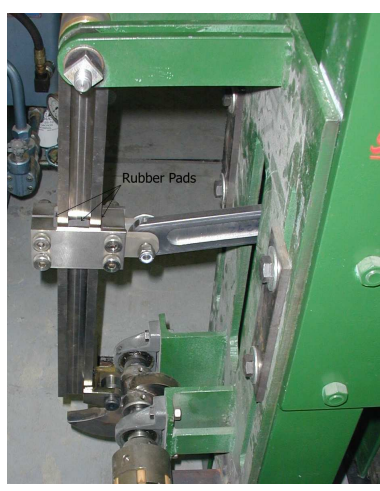

Figure 16: A photo of the Lever arm assembly [12].

green) with a spring constant $(\kappa)=124.717 \mathrm{~N} / \mathrm{mm}$ [13] was used. The coupling was positioned on the vertical arm in such way that the spring compression within the efficient operating range (15\%-25\% of the free length) could be achieved [13]. The spring was compressed for 2.36" or $60.00 \mathrm{~mm}$, which is $19.66 \%$ of its free length (12" or 304.80 $\mathrm{mm})$ [12].

Test Results and Validation: A high speed camera with 1000 frames/sec was used to measure speed at the impact. The speed at the impact was found to be equal to $6.0 \mathrm{~m} / \mathrm{sec}$ for a compression of $60.00 \mathrm{~mm}$, which compared well with that calculated from theory for the same compression. Speed at the impact was calculated using equation 15 was found to be equal to $6.88 \mathrm{~m} / \mathrm{sec}$, which overestimates the observed impact speed by $12.80 \%$, which is a reasonable error [12].

\section{Summary and Conclusions}

The version II of the impact wear tester has been designed, developed and validated in this research work. The version II of the impact wear tester has the following important features [12]: (1) It is quite lighter in weight and has fewer number of parts than the version I of the impact wear tester. (2) It can allow easy alteration of spring compression amplitude. (3) It can allow to determine impact wear rate at a wide range of impact energies. (4) It needs little or no maintenance because of its robust design. (5) It is well optimized for achieving maximum speed at the impact. (6) It can be tested for energy levels up to equivalent of $620 \mathrm{~cm}$ of drop height.

\section{Acknowledgments}

This work is co-sponsored by AMIRA-JKMRC P9M project and a Natural Sciences and Engineering Research Council (NSERC) of Canada collaborative research and development matching grant. 


\section{Symbols and Abbreviations}

\section{Latin Symbols}

$A$ : Area; $c$ : Distance from neutral axis to outer fiber associated with beam analysis; $d$ : Unit, angular; $D$ : Spring displacement; $e$ : Gross longitudinal; $E$ : Energy; $E_{y}$ : Young's modulus; $f$ : Deflection; $F$ : Force; $i$ : Slope; $I$ : Rectangular moment of inertia; $I_{p}$ or $J$ : Polar moment of inertia; $l$ : Length; $L$ : Distance between beam supports; $m$ : Mass lost associated with the wear mechanisms; $M_{b}$ : Bending moment; $M_{o}$ : Moment about fulcrum; $M_{t}$ : Torsion; $N$ : Factor of safety; $P$ : Concentrated load; $P_{a}$ : Pressure; $P_{c}$ : Probabilistic constant; $r$ : Radius; $s$ : Unit, longitudinal; $s$ ': Unit, lateral; $S$ : Unit normal apparent stress; $S_{s}$ or $S_{v}$ : Transverse shearing unit stress; $t$ : Time; $T$ : Torque; $v$ : Velocity; $V$ : Shear Stress; $w$ : Distributed load per longitudinal unit; $W$ : Total distributed load; $W^{\prime}$ : Weight of the body; $x$ : Displacement; $Z$ : Horizontal shearing stress.

\section{Greek Symbols}

$\alpha$ : Unit, angular; $\gamma_{S}$ : Coefficient of variation; $\theta$ : Angle; $\kappa$ : Spring constant; $\lambda$ : Correction factor; $\mu$ : Coefficient of friction; $\mu_{p}$ : Poisson's ratio; $\rho$ : Metal density; $\phi$ : Angular displacement.

\section{References}

[1] P. Radziszewski, "Predictive Model For Ball Mill Wear", Canadian Metallurgical Quarterly, 1997a, Vol. 36, No. 2, pp. 87-93.

[2] P. Radziszewski and S. Tarasiewicz, "Modelling and Simulation of Ball Mill Wear", Wear, 1993a, Vol. 160, pp. 309-316.

[3] P. Radziszewski and S. Tarasiewicz, "Simulation of Ball Charge and Liner Mill”, Wear, 1993b, Vol. 169, pp. 77-85.

[4] P. Radziszewski, Exploring Total Media Wear, Mechanical Engineering Department Technical Report, McGill University, Montreal, Quebec, 2001.

[5] P. Radziszewski, Chapter 4: Ball Mill Media Wear and Prediction, JKMRC/AMIRA P9L Technical Project Report, Queensland, Australia, 1997b, pp. 35-51.

[6] Blickensderfer and Tylczak, "Evaluation of Commercial US Grinding Balls by Laboratory Impact and Abrasion Tests", Miner. Metall. Process, 1989, Vol. 6, pp. 60-66.

[7] Xu, Tan and Zhou, "Impact Wear Mechanisms of Medium Carbon Steel Under Various Dry and Wet Conditions", The ASME J. of Wear of Materials, 1991, pp. 33-40.

[8] Scieszka and Dutkiewicz, "Testing Abrasive Wear in Mineral Comminution", The International J. of Mineral Processing, 1991, Vol. 32, pp. 81-110.

[9] Dorlot, Baïlon and Masounave, "Des Matériaux, Éd. l'École Polytechnique, Montréal, Quebec, 1986.

[10] P. Radziszewski, Exploring Total Media Wear, JKMRC/AMIRA P9L Technical Project Report, Queensland, Australia, 1999.

[11] P. Radziszewski, An Update on Mill Media Impact Test Improvement, JKMRC/AMIRA P9L Technical Project Report, Queensland, Australia, 2000.
[12] L. K. Thakur, Design and Development of an Impact Wear Testing Device for the Minerals Industry, Master of Engineering Thesis, Mecahanical Engineering Department, McGill University, Montreal, Quebec, 2004.

[13] Producto, The Complete Catalogue of Springs for All Your Needs, Producto Diemakers Suppliers Limited, Montreal, Quebec, 1996, pp. 1-6.

[14] M. Girard, Simulateur D'Impact. Rapport De Conception, Université du Québec en Abitibi-Témiscamingue, Montréal, Quebec, 2000.

[15] H. P. Greenspan and D. J. Benney, 1973, Calculus. An Introduction to Applied Mathematics, McGraw-Hill, Inc., New York, 1973.

[16] ADAMS/Solver, Using ADAMS/Solver, Automatic Dynamic Analysis of Mechanical Systems, MSC Software, Santa Ana, CA, 2001.

[17] ADAMS/View 12.0 Help, Intranet HTML Help On Using ADAMS/View 12.0, Automatic Dynamic Analysis of Mechanical Systems, MSC Software, Santa Ana, CA, 2001.

[18] S. D. Henry, G. M. Davidson, S. R. Lampman, F. Reidenbach, R. L. Boring and W.W. Jr. Scott, Fatigue Data Book: Light Structural Alloys, ASM International, OH, 1995, pp. 181-385 \& pp. 1-110.

[19] J. E. Shigley and C. R. Mischke, Mechanical Engineering Design, Sixth Edition, McGraw Hill, Inc., New York, 2001.

[20] R. C. Rice, B. N. Leis and D. V. Nelson, Fatigue Design Handbook/prepared under the auspices of the Design Handbook Division of the SAE Fatigue Design and Evaluation Technical Committee, AE-10, Second Edition, Society of Automotive Engineers, Warrendale, PA, 1988.

[21] T. Baumeister, E. A. Avallone and T. BaumeisterIII, Mark's Standard Handbook for Mechanical Engineers, Eighth Edition, McGraw-Hill Book Company, New York, 1988, pp. 5.25.37 .

[22] V. Adams and A. Askenazi, Building Better Products With Finite Element Analysis, OnWord Press, Santa Fe, NM, 1999.

[23] MetWeb, Material Property Data, Automation Creations, Inc., Blacksburg, VA, 2003, URL: http://www.matls.com/index.asp?ckck=1.

[24] J. Fernandes, Technical Service \& Sales Representative, Private Consulting, Elimetal, Inc., St. Laurent, Quebec, 2003.

[25] Smith Fasteners, Socket Shoulder Screws Dimensions and Specifications, Smith Fastener Company, Bell, CA, 2003, URL: http://www.smithfast.com/shoulderscrew.html.

[26] Titanium Industries, Data \& Reference Manual, Titanium Industries, Inc., Montreal, QC, 2003. 